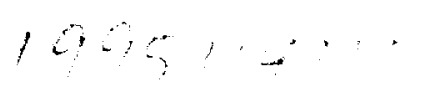

\title{
PARASITIC CURRENT COLLECTION BY SOLAR ARRAYS IN LEO ${ }^{1}$
}

\author{
Victoria A. Davis and Barbara M. Gardner \\ S-Cubed Division of Maxwell Laboratories \\ San Diego, California
}

\begin{abstract}
SUMMARY
Solar cells at potentials positive with respect to a surrounding plasma collect electrons. Current is collected by the exposed high voltage surfaces: the interconnects and the sides of the solar cells. This current is a drain on the array power that can be significant for high-voltage arrays. In addition, this current influences the current balance that determines the floating potential of the spacecraft. One of the objectives of the Air Force (PL/GPS) PASP Plus experiment is an improved understanding of parasitic current collection. As part of the PASP Plus program, we are using computer modeling to improve our understanding of the physical processes that control parasitic current collection.
\end{abstract}

\section{BACKGROUND}

Solar arrays provide power for nearly all space systems. Traditionally, solar arrays have operated in the $30 \mathrm{~V}$ range to avoid complex interactions with the plasma environment. As space systems become more complex, more power, therefore higher voltages, is needed.

Typically, the negative side of each solar array is grounded to the spacecraft chassis. This makes the exposed metal and semiconductor of the arrays positive with respect to the spacecraft body. The equilibrium potential of a spacecraft with respect to the plasma is the potential at which there is no net current to the spacecraft. As illustrated in figure 1, there are several components of the current to a spacecraft. Portions of the solar array attract ions and other portions attract electrons, depending on the local potential. Ions are attracted to exposed conductors on the spacecraft surface, as the spacecraft body is negative with respect to the plasma. Particle beams and other emitters also contribute to the net current. Since electrons are more mobile than ions, spacecraft float negative unless the potential is actively controlled.

It may be necessary to keep the spacecraft body near zero potential with respect to the plasma. For example, an instrument to measure the low energy plasma environment may need to be near plasma ground. Anodization arcing and negative potential arcing are potentially disruptive at potentials greater than 50 to $100 \mathrm{~V}$ negative with respect to the plasma (refs. 1 and 2). Particle beams and plasma contactors are sometimes used to maintain the spacecraft potential. If the solar arrays are at $150 \mathrm{~V}$ and the spacecraft chassis is maintained at no more than $50 \mathrm{~V}$ negative with respect to the plasma, the most positive portions of the solar arrays are at $100 \mathrm{~V}$, with respect to the plasma. Therefore, it is important to consider current collection by the portions of solar arrays at positive potentials.

A traditional solar array is an array of solar cells 2 to $8 \mathrm{~cm}$ in size and connected in series by millimeter-sized metal interconnects. The interconnects can be metallic meshes or sets of wires. Each solar cell is protected from the environment by a cover glass. Figure 2 shows the regions between cells in a typical array. Current is collected by the portions of the metallic interconnects and the semi-conducting solar cells exposed to the plasma environment.

${ }^{1}$ This work is supported by the Air Force Materiel Command. 
At low potentials, a barrier forms that prevents electrons from reaching the high voltage surfaces. The barrier is created by the surrounding insulating surfaces, primarily the coverglass, that float slightly negative. The current collected is the high energy tail of the distribution and therefore depends exponentially on the barrier height. The barrier height depends on the potential, the plasma, and the geometry.

At high potentials, typically over $200 \mathrm{~V}$, the current rapidly rises due to a phenomena called snapover. Snapover was first observed at NASALeRC (refs. 3, 4, and 5). Snapover can occur whenever there is a biased surface adjoining an insulating surface and the bias is above the first crossover of the secondary yield curve of the insulating surface (refs. 6 and 7 ).

When the solar cell potential is below the first crossover of the secondary yield curve, the insulating surface floats slightly negative (a few times the electron energy) and collects as many ions as electrons from the ambient plasma. If the potential of the insulating surface is above the first crossover, each electron generates more than one electron. The secondary electrons may either return to the surface or move across the surface under the influence of surface electric fields. The equilibrium potential at each location is the potential at which the net current of electrons from the plasma and secondary electrons arriving at and leaving the location balance. The exposed conductor is a sink for the secondary electrons. The surfaces adjust so that a potential gradient exists across the insulating surface attracting the secondaries to the conductor at the highest potential. The net effect is that the high potential area and the collecting area increase. There is a range of applied bias values for which the insulating surface may either float slightly negative or be snapped over. Experimentally, hysteresis is observed. The size of the snapped over region, and therefore the current, depends on the local geometry as well as the potentials and the plasma.

The Photovoltaic Array Space Power Plus Diagnostics (PASP Plus) flight experiment will explore high voltage current collection by solar arrays (ref. 8). PASP Plus is the principal experiment integrated onto the Advanced Photovoltaic and Electronics Experiments (APEX) satellite bus. APEX will be launched this summer into a $70^{\circ}$ elliptical orbit from 360 to $1950 \mathrm{~km}$. The spacecraft attitude is such that the solar array test panels will always face the sun. The experiment will test twelve different solar array designs. The experiment will investigate negative potential arcing, parasitic current collection, and long term radiation damage. Parasitic current collection will be measured for eight of the designs under various operational and environment conditions. The arrays will be biased from 75 to $400 \mathrm{~V}$. Previous space experiments that examined parasitic current collection include PIX I (ref. 9), PIX II (refs. 10 and 11) and SAMPIE (ref. 12).

\section{OUR APPROACH}

The computation of the current collected by a specific solar array can become intractable. The gap size is of the order of tens of mils while the solar cells are a few centimeters and the entire array can be meters. Each solar cell is at a slightly different potential. The current depends on the geometry of the gap, the geometry of the entire array, the spacecraft, and the plasma conditions.

We are interested in improving our understanding of which aspects of the problem are most important and developing a tool or at least an algorithm to assist spacecraft designers. Our approach is to look in detail at current collection at a single cell gap. Using the computer we can vary each parameter independently. We then develop formulas that estimate the current collected by a single gap. We then incorporate the formulas into a tool that can add up the current from all the gaps to give the current collected by an array. Information on the array geometry and how it influences the current can also be added to the tool.

To compute the current to a single gap, we are using the Gilbert computer code. Gilbert is a generalpurpose, two-dimensional, plasma analysis code. It can be used to solve for the electrostatic potential about an object, with flexible boundary conditions on the object and with space charge computed either 
fully by particles, fully analytically, or in a hybrid manner. For this study Gilbert is used to first compute the electrostatic potentials in space around the solar cells and then to compute the electron trajectories in the previously computed potentials. The space charge was computed using an analytic formulation. The analytic formula includes charge density variation due to acceleration and convergence (ref. 13).

Figure 5 shows a typical grid for the Gilbert calculations. The gap and the space above the gap are gridded. The surfaces provide the boundary conditions. This grid represents solar cells with mesh interconnects. An idealized geometry was chosen because we are interested in understanding the relationship between the variables. The interconnect is modeled as a diagonal line extending across the bottom of the gap. The side of one solar cell is at a high potential along the left side of the gap. The sides of the coverglass on both sides of the gap are included. All the insulating surfaces have a special boundary condition that represents the snapover condition when appropriate.

Figure 6 shows potential contours and a few trajectories for a typical case. The potentials are computed using an analytic representation of the charge density. Macroparticles are emitted from several locations along each sheath segment. At each location, a set of particles with a thermal distribution of velocities is created. These macroparticles are tracked. The collected current is the current carried by the macroparticles reaching the high potential surfaces.

Current collection can be either orbit limited or space charge limited, depending on the debye length of the plasma and the size of the collecting area.

There are two serious limitations to computing the current in this way. Implicit in the technique of drawing a sheath edge and tracking current from this sheath edge is the assumption of a sharp sheath edge. This assumption is not necessarily valid when the sheath size is of the order of a debye length. The computed sheath sizes are smaller than a debye length for some geometries under some plasma conditions, particularly for geometries without an interconnect and at low potentials.

The other limitation is the assumption that current can reach the sheath. At lower cell potentials, the coverglass surface potential dominates the long distance potential. To reach the "sheath edge," an electron must pass through a negative potential region. Only the electrons in the high energy tail of the distribution are collected. A different technique is needed to compute the current in this regime.

A different approach is also needed at the highest potentials. When the entire surface of the array is snapped over, the sheath is dominated by three-dimensional effects.

\section{RESULTS}

We have done calculations for three geometries: cell-to-cell gap region with an interconnect, without an interconnect, and a single cell edge. The calculations span the space of anticipated plasma conditions, applied potential, and first crossover potential. The current rises rapidly when the applied bias is four to five times the first crossover potential. Figure 7 shows how the current varies as a function of the applied bias for a single solar cell edge.

We now have analytic formulas for the dependence of the current on the primary problem variables: applied voltage, plasma conditions, and, to some extent geometry. These formulas have been incorporated into the EPSAT computer code.

EPSAT is an analysis tool for determining the performance of power systems in both naturally occurring and self-induced environments (ref. 14). EPSAT is an engineering spreadsheet that allows rapid "what if" analysis of the effect of parametric changes on a space-based system's performance. EPSAT provides information on the plasma and neutral environment anticipated on orbit. The code presently models many of the interactions of the space environment with a power system, including sheath 
formation, $v \times$ B potentials, particle beams, and sheath ionization. The code permits the user to do mission studies and evaluate the importance of the various interactions with a specific spacecraft design. The user can then adjust the design and examine the effect of the change on the ability of the system to operate in natural or hostile environments. EPSAT uses a unique architecture for integrating analysis models that allows modeling capabilities to evolve with changing needs.

A new solar array module for EPSAT has been developed. The current to a solar array is computed by summing the current to each solar cell edge, interconnect, and gap without interconnect, while not double counting. The current to a single edge or gap is computed using a fit to the two-dimensional computations.

Figure 8 shows the current to PASP Plus array \#1, mesh interconnect design, under typical space conditions for different values of the first crossover potential. Figure 9 shows the current to PASP Plus array \#3, space station design, under typical space conditions for different values of the first crossover potential.

Figure 10 shows how the current computed in this manner compares with laboratory measurements made on the PASP Plus array \#8. Array 8 has a wrap-thru interconnect design.

\section{CONCLUSIONS}

We are refining our two-dimensional calculations and examining their limitations. As needed, we will use other techniques to extend the range of our calculations. The results are continuing to be incorporated into EPSAT. We look forward to comparing the flight measurements with the calculations. Once our understanding is validated, the formulas developed can be used to improve the design of solar cells to minimize complications due to this interaction.

\section{REFERENCES}

1. Vaughn, J.A., et al.: Extrapolation of Electrical Breakdown Currents from the Laboratory to Space Station. AlAA 92-0822, AlAA 30th Aerospace Sciences Meeting, Reno, Nevada, 1992.

2. Ferguson, D.C.: The Voltage Threshold for Arcing for Solar Cells in LEO - Flight and Ground Test Results. NASA TM 87259, 1986.

3. Purvis, C.K.; Stevens, N.J.; and Berkopec, F.D.: Interaction of Large High Power Systems with Operational Orbit Charged-Particle Environments. NASA TM X-73867, 1977.

4. Stevens, J.N.: Interactions Between Spacecraft and the Charged-Particle Environment. Spacecraft Charging Technology - 1978, NASA Conference Publication 2071, AFGL-TR-79-0082, 1978, pp. 268-294.

5. Kennerud, K.L.: High Voltage Solar Array Experiments, NASA Rep. CR-121280, 1974.

6. Chaky, R.C.; Nonnast, J.H.; and Enoch J.: Numerical Simulation of Sheath Structure and CurrentVoltage Characteristics of a Conductor-dielectric Disk in a Plasma. J. Appl. Phys., vol. 52, 1981, pp. 7092-7098.

7. Katz, I.; and Mandell, M.J.: Differential Charging of High-Voltage Spacecraft: The Equilibrium Potential of Insulated Surfaces. J. Geophys. Res., vol. 87, 1982, pp. 4533-4541. 
8. Guidice, D.A.: PASP Plus: An Experiment to Measure Space-Environment Effects on Photovoltaic Power Subsystems. Fifth Annual Workshop on Space Operations Applications and Research (SOAR '91), NASA Conference Publication 3127, vol. II, 1991, pp. 662-668.

9. Grier, N.T.; and Stevens, N.J.: Plasma Interaction Experiment (PIX) Flight Results. Spacecraft Charging Technology - 1978, NASA Conference Publication 2071, AFGL-TR-79-0082, 1979. pp. 295-314.

10. Grier, N.T.: Plasma Interaction Experiment II (PIX II): Laboratory and Flight Results. Spacecraft Environmental Interactions Technology, 1983 , NASA Conference Publication 2359, AFGL-TR-850018,1985, pp. 333-347.

11. Ferguson, D.C.: RAM/WAKE Effects on Plasma Current Collection of the PIX II Langmuir Probe. Spacecraft Environmental Interactions Technology, 1983, NASA Conference Publication 2359, AFGL-TR-85-0018, 1985, pp. 349-357.

12. Hillard, G. B.; and Ferguson D.C.: Solar Array Module Plasma Interactions Experiment (SAMPIE): Science and Technology Objectives. J. of Spacecraft and Rockets, vol. 30, 1993, pp. 488-494.

13. Mandell, M.J.; and Davis, V.A.: User's Guide to NASCAP/LEO. LEW-15641, SSS-R-85-7300-R2, 1990. (Available as LEW-15641 from COSMIC, U of Georgia.)

14. Kuharski, R.A., et al. High Voltage Interactions of a Sounding Rocket with the Ambient and SystemGenerated Environments. IEEE Transactions on Nuclear Science, vol. 37, 1990, pp.2128-2133.

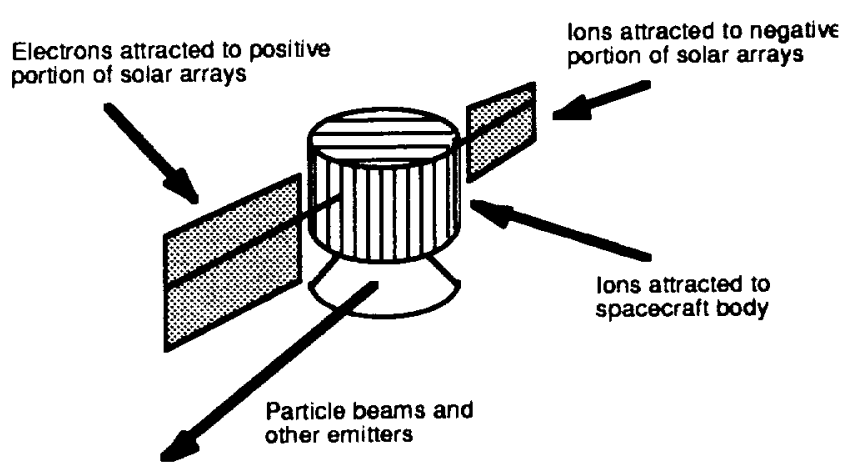

Figure 1.-At the spacecraft floating potential the net current is zero.

This current has several components.

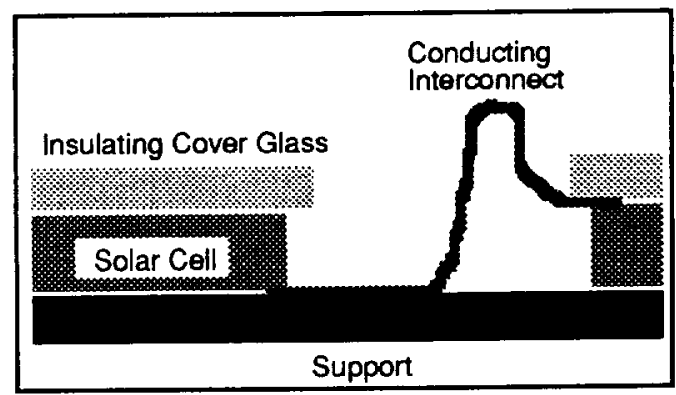

Figure 2.-Cross section of the region between traditional solar cells. 


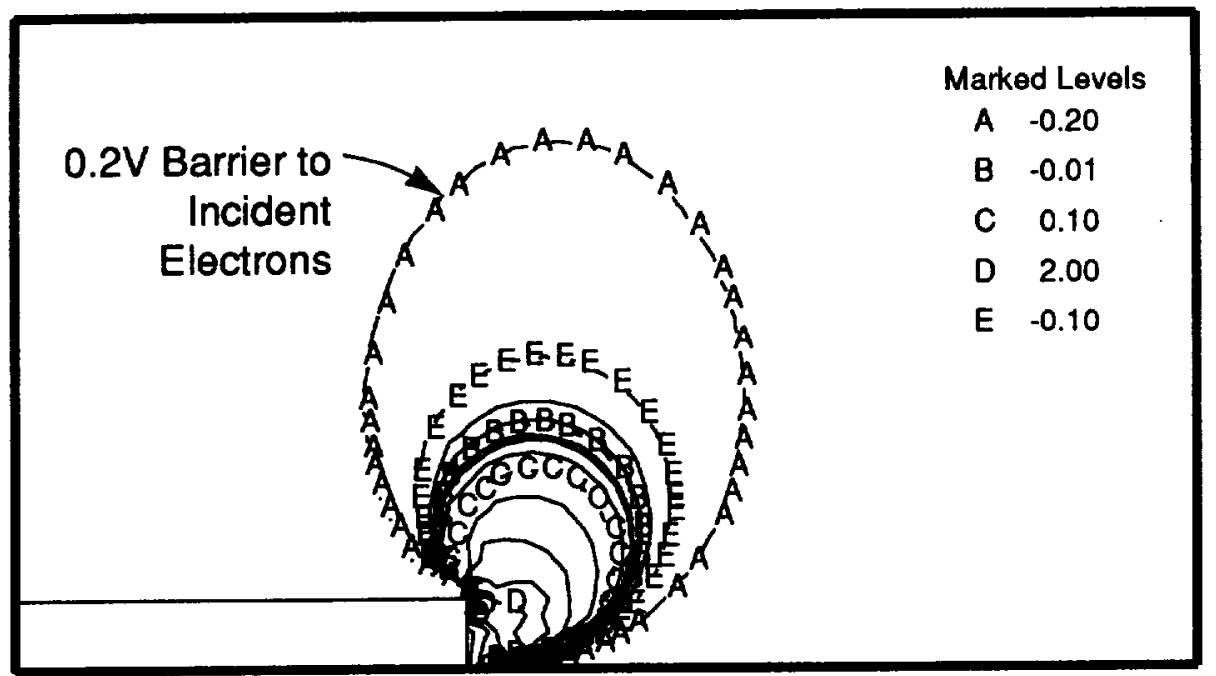

Figure 3.-At low potentials, barrier formation dominates current collection. Plasma of $10^{12} \mathrm{~m}^{-3}$ and $0.1 \mathrm{eV}$. Cell potential of $25 \mathrm{~V}$, surface potential of $-0.1 \mathrm{~V}$. First crossover above $25 \mathrm{~V}$.

No Snapover:

dielectric potential $\sim 0$

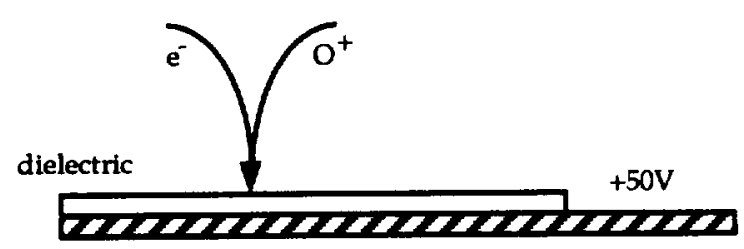

conductor
Dielectric surface collects as many ions as electrons from the ambient plasma.
Snapover:

dielectric potential $>\mathrm{E}$

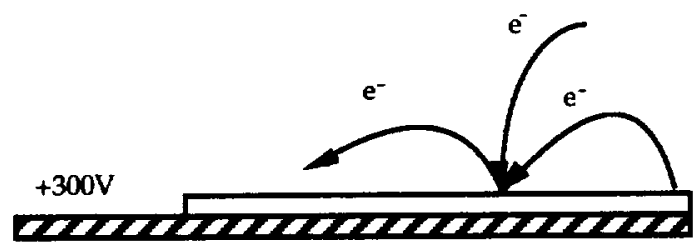

Dielectric surface emits as many secondary electrons as it collects primaries and secondaries.

Figure 4.-When a conductor is at a potential above the first crossover of the secondary yield curve of an adjoining insulator, the insulator surface can develop positive potentials in a phenomena known as snapover. 


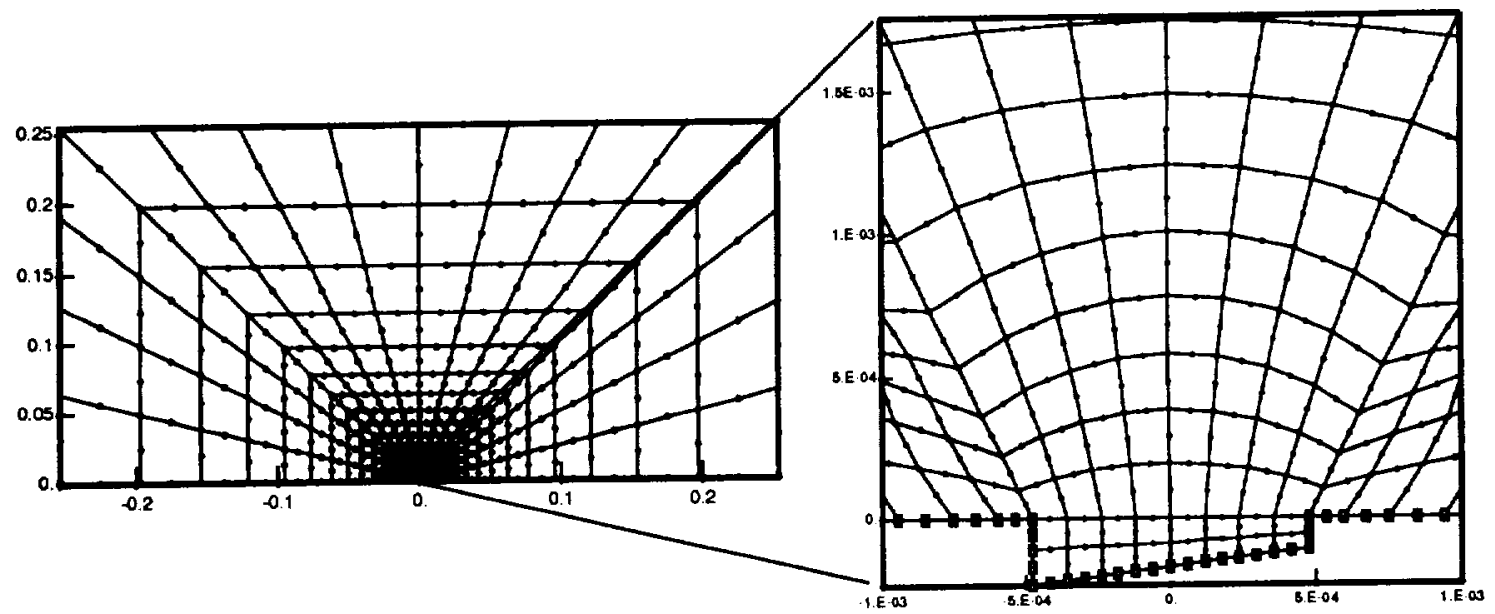

Figure 5.-Girid for two-dimensional Gilbert calculations.

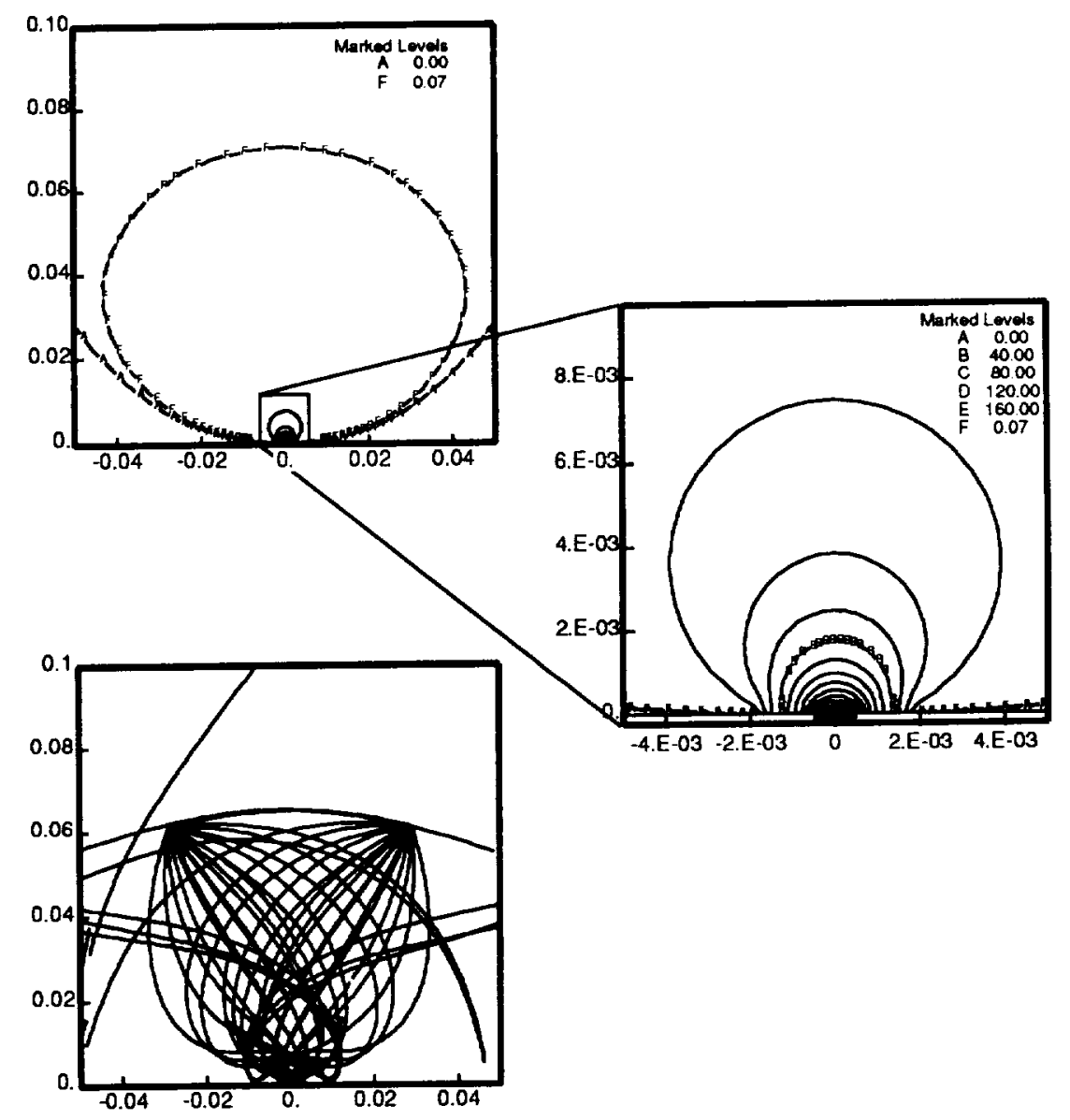
Figure 6.-Potential contours and a few representative trajectories. Plasma of $10^{11} \mathrm{~m}^{-3}$ and $0.1 \mathrm{eV}$.
Cell potential of $150 \mathrm{~V}$, surface potential of $-0.5 \mathrm{~V}$, first crossover of $40 \mathrm{~V}$. 


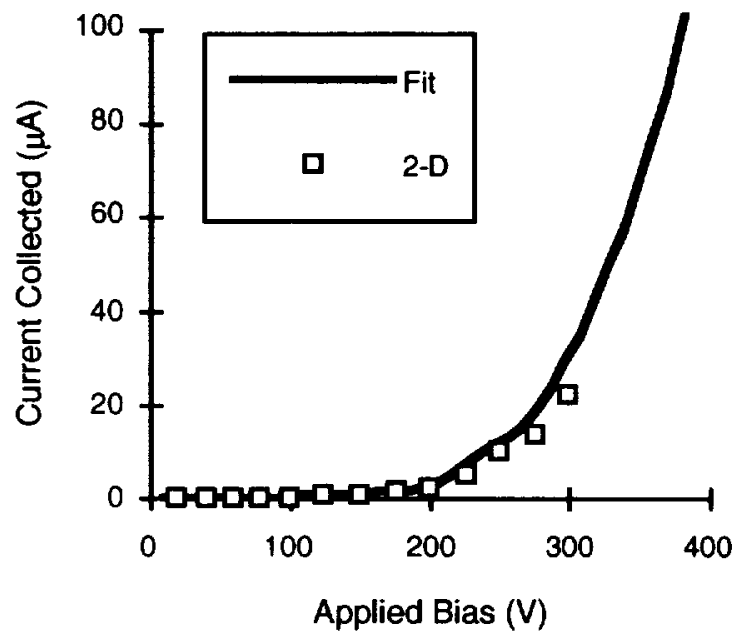

Figure 7.-Current to $1 \mathrm{~m}$ along $5 \mathrm{mil}$ thick solar cell edges as a function of applied bias. Plasma of $10^{10} \mathrm{~m}^{-3}$ and $0.1 \mathrm{eV}$. First crossover of $40 \mathrm{~V}$ and surface potential of $-0.5 \mathrm{~V}$. The points are the results of Gilbert calculations and the line is a fit.

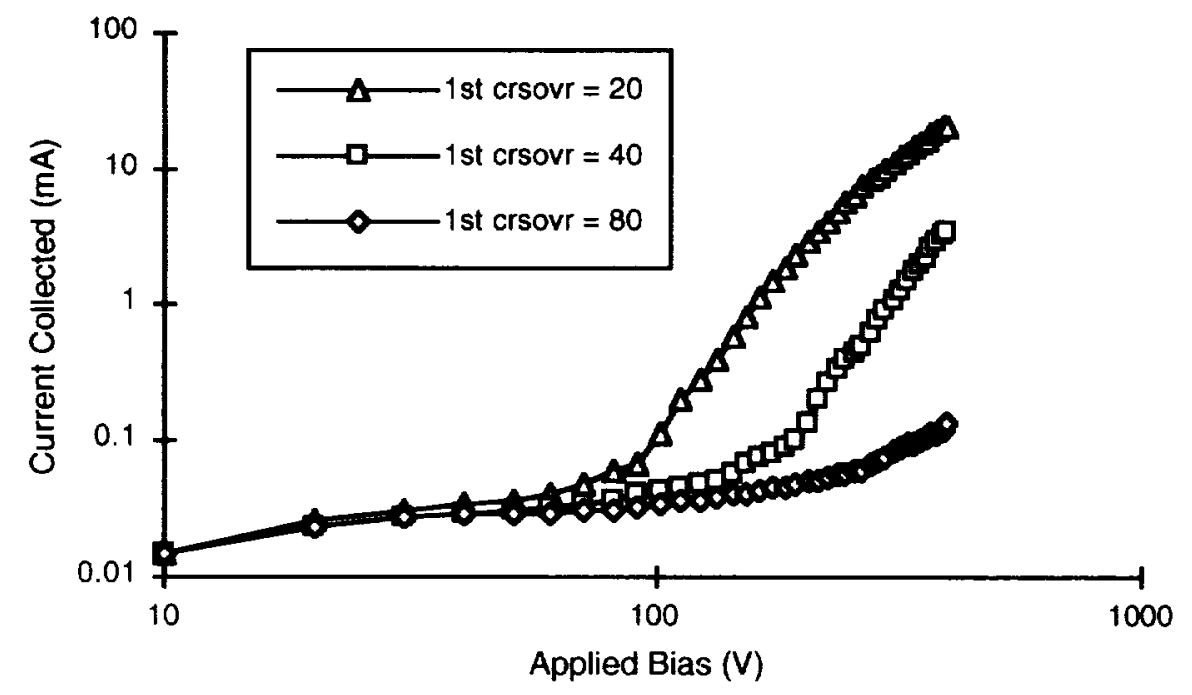

Figure 8. - Current to PASP Plus array \#1, mesh interconnect design, as a function of applied bias. Plasma of $1.9 \times 10^{11} \mathrm{~m}^{-3}$ and $0.088 \mathrm{eV}$. Surface potential of $-0.5 \mathrm{~V}$. Three value of the first crossover of the secondary yield. 


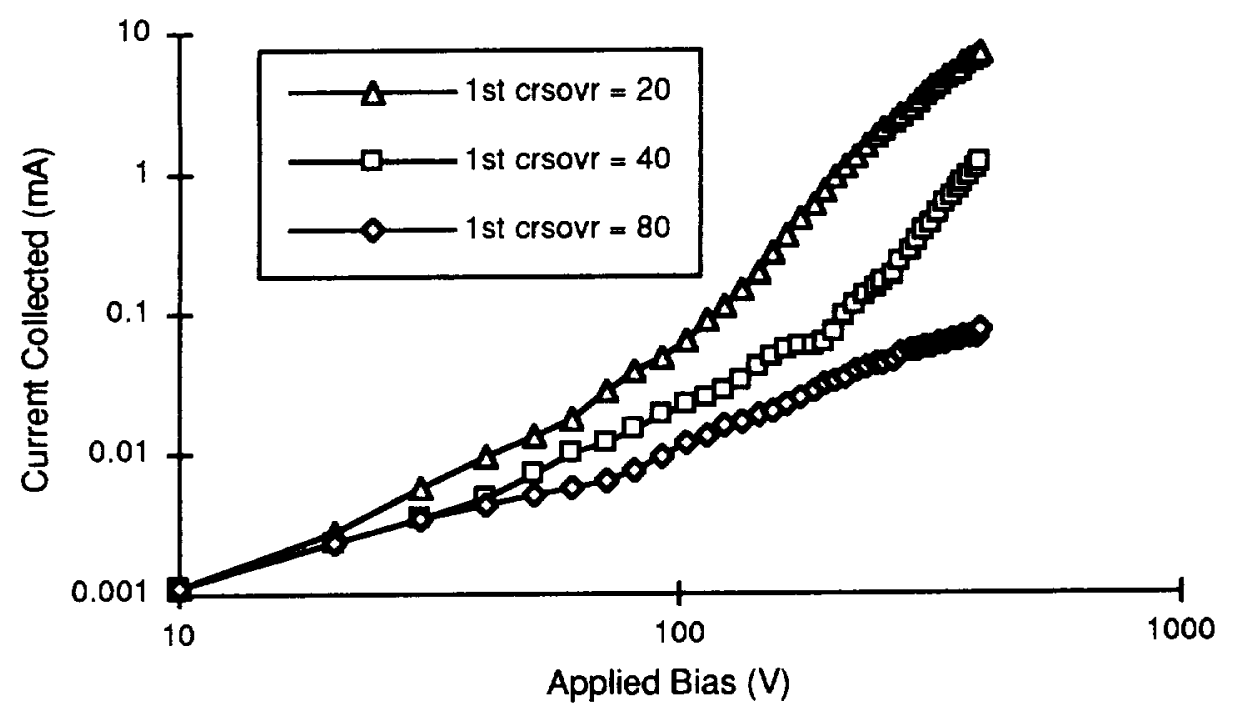

Figure 9.-Current to PASP Plus array \#3, space station design, as a function of applied bias. Plasma of $1.9 \times 10^{11} \mathrm{~m}^{-3}$ and $0.088 \mathrm{eV}$. Surface potential of $-0.5 \mathrm{~V}$.

Three value of the first crossover of the secondary yield.

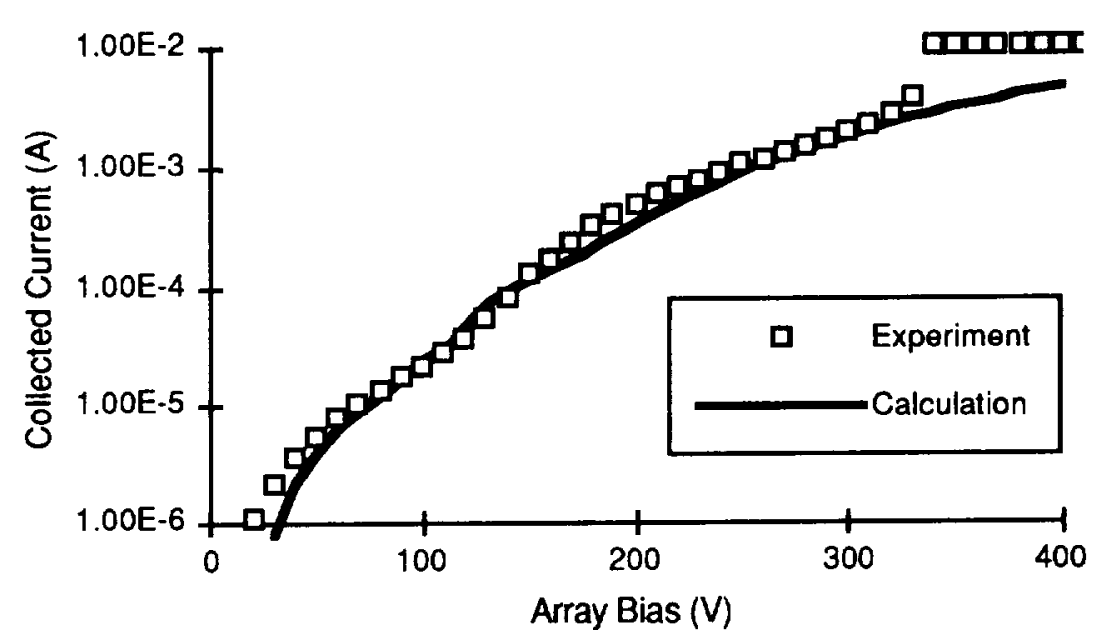

Figure 10.-Comparison of model of parasitic current collection with laboratory measurements for PASP Plus array \#8. The environment is $4 \times 10^{11} \mathrm{~m}^{-3}$ at $0.5 \mathrm{eV}$.

The first crossover potential is assumed to be $25 \mathrm{~V}$.

(Unpublished experimental data courtesy of N. T. Grier of NASALeRC.) 



\section{SESSION IV}

\section{FLIGHT RESULTS}


